\title{
Stability \& Chaos Tests of 2D Discrete Time Dynamical System with Hidden Attractors
}

\author{
Maysoon M. Aziz, Omar M. Jihad \\ Department of Mathematics, College of Computer Science and Mathematics, University of Mosul, Mosul, Iraq \\ Email: aziz_maysoon@yahoo.com,omarmoh1992h@gmail.com, aziz_maysoon@uomosul.edu.iq
}

How to cite this paper: Aziz, M.M. and Jihad, O.M. (2021) Stability \& Chaos Tests of 2D Discrete Time Dynamical System with Hidden Attractors. Open Access Library Journal, 8: e7501.

https://doi.org/10.4236/oalib.1107501

Received: May 7, 2021

Accepted: June 20, 2021

Published: June 23, 2021

Copyright $\odot 2021$ by author(s) and Open Access Library Inc.

This work is licensed under the Creative Commons Attribution International License (CC BY 4.0).

http://creativecommons.org/licenses/by/4.0/

(c) (i) Open Access

\begin{abstract}
In this research two-dimensional dynamical system was taken. The system was analyzed through its fixed points, stability analysis, chaos diagnoses and adaptive control technique. It was found that the system had a hidden attractor and unstable fixed points. The largest value of Lyapunov exponent equals 1.853981 and from binary test we get: $k=0.9305$, which indicates that the system is chaotic. Adaptive control technique was performed, and it was found that the system is stable and regular after control.
\end{abstract}

\section{Subject Areas}

Dynamical System

\section{Keywords}

Hidden Attractors, Chaos, Lyapunov Exponent, Lyapunov Dimension, Binary Test, Adaptive Control

\section{Introduction}

In recent years, we have seen that there is great interest in the subject of hidden and self-Excited attractors, the attractors in chaotic dynamical systems are classified into two categories: dynamical systems with self-Excited attractors and dynamical systems with hidden attractors, as it is said for the systems whose basin of attraction intersects with the neighborhood. The Lorenz system [1] is one of the chaotic systems that have self-Excited attractors, which was proposed in 1963, while the systems in which the basin of attraction does not intersect with the open neighborhood of equilibrium and which does not have fixed points or equilibrium or it has only one stable fixed point or nodal points, so it is called a dynamical system with hidden attractors. Jerk-like system [2] is one of the systems that have hidden attractors. There are many researchers who have provided 
a number of models in dynamical systems that have these types of attractors, Jafari and Sprott [3] managed to create a list of 17 chaotic models with nonlinear quadratic boundaries that have no equilibrium points, which were classified by Leonov and Kuznetsov [4] [5] as systems with hidden attractors, and Chan and Wang introduced an anarchic system with only one stable point equilibrium [6] through some modifications to Sprott system [7], and Wei [8] designed a fourdimensional system, which turned out to be a hyper-chaotic system without equilibrium points.

Models in dynamical systems have hidden attractors for many applications, especially in the field of engineering designs, because they do not allow for unforeseen accidents [7] such as potential disturbances in the structure of bridges and aircraft wings [9] and others. This paper was arranged as follows: system description, system analysis and finding fixed points, stability analysis of fixed points using stability tests: characteristic roots test, jury test [10] [11], study of the dynamical behavior of the system, finding phase space and bifurcation diagrams [12] [13] and Chaos diagnosing using chaos tests: the Lyapunov exponent test and Lyapunov dimension [14] [15], binary (0 - 1) test [16] [17]. The problem of chaos control attract attention of mathematician, researchers and engineers and there are many practical reasons for controlling chaos one of them chaos causes irregular behavior in nonlinear dynamical systems, therefor chaos control should be eliminated as much as possible or totally suppressed, adaptive control technique [18] [19] were used to control.

\section{System Description}

Two-dimensional discrete time dynamical system, suggested by Panahi [20] [21] and the define is as follows:-

$$
\begin{gathered}
F=\left\{\begin{array}{l}
x_{t+1}=x_{t}+y_{t} \\
y_{t+1}=0.1 x_{t}^{2}+0.1+y_{t}\left(-x_{t}-b y_{t}\right)+y_{t}
\end{array}\right. \\
\quad b=2
\end{gathered}
$$

where $x_{t}, y_{t}$ represent variables system and $b$ represent parameter system.

\section{System analysis}

In this section analysis the system was done by means of its fixed points. Let us assume that:-

$$
\begin{aligned}
& f_{1}\left(x_{t}, y_{t}\right)=x_{t} \\
& f_{2}\left(x_{t}, y_{t}\right)=y_{t}
\end{aligned}
$$

To find the fixed points of system (1), suppose that:

$$
\begin{gathered}
y_{t}=0 \\
0.1 x_{t}^{2}+0.1+y_{t}\left(-x_{t}-b y_{t}\right)+y_{t}=y_{t}
\end{gathered}
$$

By solving Equations (3) and (4), we obtain the following points:- 


$$
p_{0}=(i, 0), \quad p_{1}=(-i, 0)
$$

Since the obtained fixed points are nodal points, we can say that the dynamical system (1) have hidden attractors.

The Jacobian matrix of system (1) obtained as:

$$
J_{\left(x_{t}, y_{t}\right)}=\left[\begin{array}{cc}
1 & 1 \\
0.2 x_{t}-y_{t} & -x_{t}-2 b y_{t}+1
\end{array}\right]
$$

Proposition (1):- Let

$$
L(\lambda)=a_{2} \lambda^{2}+a_{1} \lambda+a_{0}=0
$$

A characteristic Equation of (5) and $\left|\lambda_{i}\right|, i=1,2$ are values of the roots of Equation (6), then the following cases are true:

1) If $\left|\lambda_{i}\right|<1$, then the fixed point of system (1) is locally asymptotically stable and is called the sink.

2) If $\left|\lambda_{i}\right|>1$, then the fixed point of system (1) is unstable and is usually called source, but if at least one of the values of the roots of Equation (6) is greater than one, then the fixed point is called Saddle.

3) If $\left|\lambda_{i}\right|=1$, then the fixed point of system (1) is called Non-Hyperbolic point, but if $\left|\lambda_{i}\right| \neq 1$, then the fixed point is called Hyperbolic point.

Proposition (2):-

From the Equation (6), then the jury Table 1 is obtain as:

Such that

$$
b_{k}=\left|\begin{array}{cc}
a_{0} & a_{n-k} \\
a_{n} & a_{k}
\end{array}\right|, k=0,1, n=2, \quad c_{k}=\left|\begin{array}{cc}
b_{0} & b_{n-1-k} \\
b_{n-1} & b_{k}
\end{array}\right|, k=0, n=2
$$

Then the fixed point of system (1) is called stable if the satisfies following conditions:

$$
L(1)>0,(-1)^{n} L(-1)>0,\left|a_{0}\right|<a_{n},\left|b_{0}\right|>\left|b_{n-1}\right|,\left|c_{0}\right|>\left|c_{n-2}\right|,
$$

where $L$ is the characteristic equation. Otherwise, the fixed point is called unstable.

\section{Stability Analysis}

In this section the stability analysis of the fixed points of the system (1) is analyzed.

Table 1. Jury table.

\begin{tabular}{lll}
\hline$\lambda^{0}$ & $\lambda^{1}$ & $\lambda^{2}$ \\
$a_{0}$ & $a_{1}$ & $a_{2}$ \\
$a_{2}$ & $a_{1}$ & $a_{0}$ \\
$b_{0}$ & $b_{1}$ & \\
$b_{1}$ & $b_{0}$ & \\
$c_{0}$ & & \\
\hline
\end{tabular}




\section{Characteristic Equation Roots Test}

We will test the stability of the fixed points of system (1) by using the test of roots characteristic equation. To test the stability of the fixed point $p_{0}$, we substitute the point $p_{0}=(i, 0)$ in Equation (5), we obtain:-

$$
J_{(i, 0)}=\left[\begin{array}{cc}
1 & 1 \\
0.2 i & -i+1
\end{array}\right]
$$

Finding the determinant $(\operatorname{Det}(\lambda I-J)=0)$, we get:-

$$
\begin{gathered}
\left|\left[\begin{array}{ll}
\lambda & 0 \\
0 & \lambda
\end{array}\right]-\left[\begin{array}{cc}
1 & 1 \\
0.2 i & -i+1
\end{array}\right]\right|=0 \\
\Rightarrow \lambda^{2}+(-2+i) \lambda+(1-1.2 i)=0
\end{gathered}
$$

By analyzing Equation (7), we get:-

$$
\lambda_{1}=1, \lambda_{2}=1
$$

From the Proposition (1) we obtain the following values:-

$$
\left|\lambda_{1}\right|=1,\left|\lambda_{2}\right|=1
$$

Then the fixed point $p_{0}=(i, 0)$ is a non-hyperbolic point, and in the same way point $p_{1}=(-i, 0)$ was tested, and its results are shown in Table 2 , which turned out to be non-hyperbolic as well.

Note (1)

In this system, we did not use Jury test to study the stability of fixed points of the system, since Jury test depends on the coefficients Equation (7) which represent complex values.

\section{Dynamical Behavior and Numerical Results of System (1)}

After studying the theoretical side of the system, we will discuss the practical side of the system, and at the beginning we will use Newton Raphson's numerical method, Newton Raphson's numerical method was used to generate the best values for the system (1) with the least possible error, as the system parameter was fixed at the value $b=2$, the following values were obtained: $(x, y)=(1.8,-0.39)$ and an error of (0.0001). Time behavior of system (1) was studied by generating a time series of system states with time $(t=100)$, shown in Figure 1 , which shows the unstable behavior of system states $x_{t}, y_{t}$ with time. The phase space was found for the variables system (1), shown in Figure 2, which shows us the paths for variables system (1) with hidden attractors. The bifurcation diagrams

Table 2. Results of the points test $p_{0}, p_{1}$ for system (1) using the characteristic equation roots test.

\begin{tabular}{ccc}
\hline Fixed points & Results of the roots of the characteristic equations of points & Status \\
\hline$p_{0}=(i, 0)$ & $\lambda_{1}=1, \lambda_{2}=1$ & Non-hyperbolic \\
$p_{1}=(-i, 0)$ & $\lambda_{1}=1, \lambda_{2}=1$ & Non-hyperbolic \\
\hline
\end{tabular}



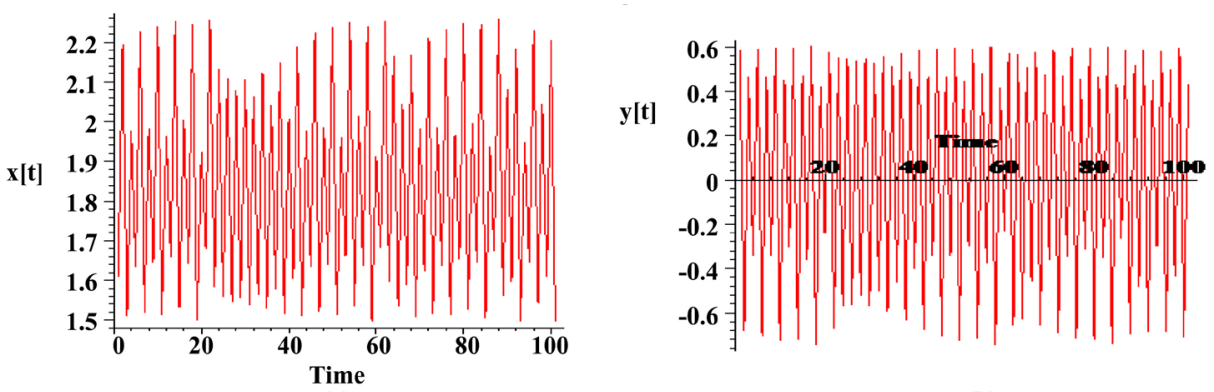

(a)

(b)

Figure 1. Time behavior of system (1). (a): $x_{t}$ versus Time; (b): $y_{t}$ versus Time.

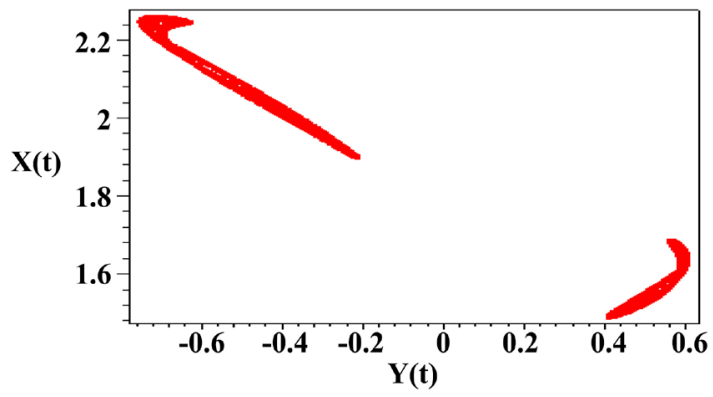

Figure 2. The phase space of variables system (1).

of the bifurcation parameter of the system (1) were found, the parameter $b$ was fixed at the interval ranging from 1.975 to 2, shown in Figure 3, which shows the bifurcation of parameter $b$ with variable $x_{t}, y_{t}$ indicating chaotic behavior of the system (1).

\section{Lyapunov Exponent and Lyapunov Dimension}

To study the chaotic behavior of system (1) we will use Lyapunov exponent test, and after applying and calculating Lyapunov exponent of system (1) the following values were obtained:-

$$
L_{1}=-0.250000, \quad L_{2}=1.853981
$$

And since one of the values of Lyapunov exponent is a positive value, this indicates that system (1) is a chaotic system, and Figure 4 shows us the chaotic behavior of system (1).

To find Lyapunov dimension, we use the following formula:

$$
p+\frac{L_{1}}{\left|L_{2}\right|}=1+\frac{-0.250000}{|1.853981|}=1-0.134844=0.865156=D_{L}
$$

\section{Binary Test}

To study and define the mess more broadly, binary test $(0-1)$ was used to determine the chaos of system (1), and by using a mathematical program in MATLAB, the system binary test was applied as a time series of system states and (1000) were generated from the iterations with a fixed value Parameter $b=2$ and $(x, y)=(1.8,-0.39)$, and $q_{c}(t)$ was calculated against $p_{c}(t)$ and shown 


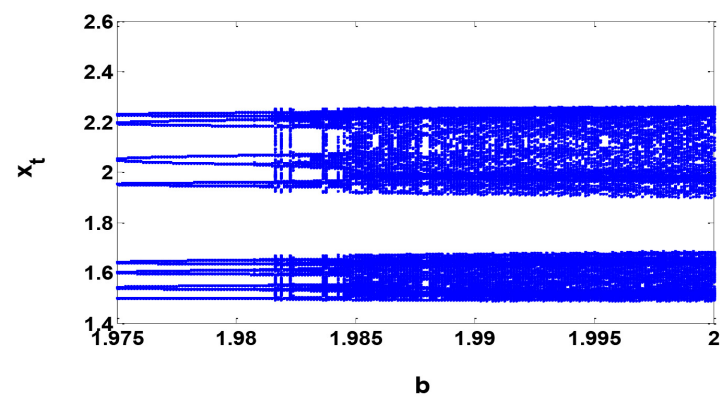

(a)

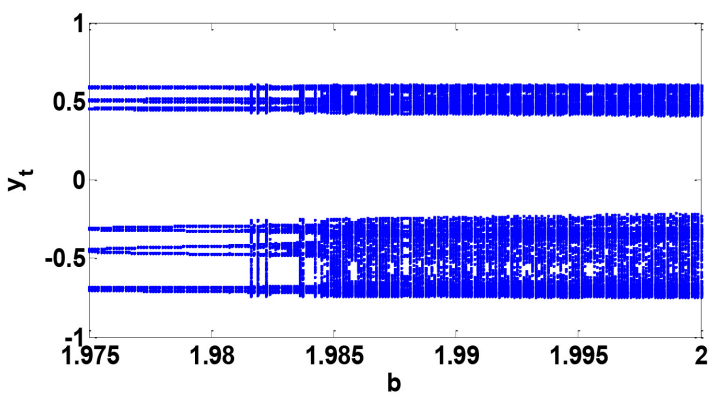

(b)

Figure 3. Bifurcation for parameter $b$ with variables system (1). (a) $x_{t}$ versus $b$; (b) $y_{t}$ versus $b$.

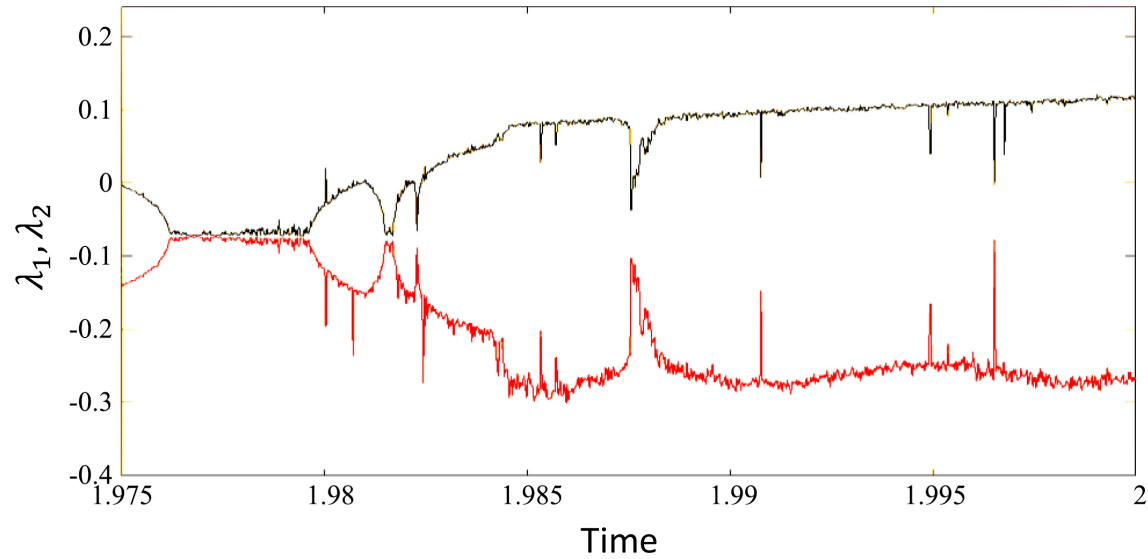

Figure 4. Lyapunov exponent of system (1).

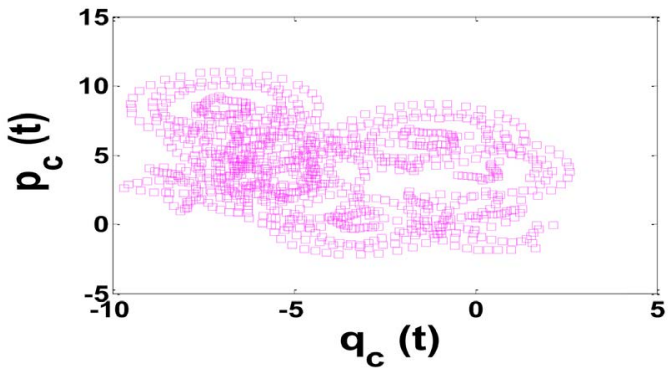

Figure 5. $p_{c}(t)$ versus $q_{c}(t)$ for system (1).

in Figure 5, which shows us a behavior similar to Brownian motion, and the mean square of the displacement $M_{c}(t)$ was found with time $(t=100)$ and shown in Figure 6, which shows the linear growth of the average square of the displacement with time, and the mean $(k)$ was found for the growth aligned with $K_{c}$ with (c) where $c \in(0, \pi)$, as it was Getting $k=0.9305 \cong 1$, which indicates chaotic of the system (1), shown in Figure 7.

\section{Adaptive Control Technique}

To address the chaos of system (1) we will use the adaptive control technique and design an adaptive control law with the unknown parameter $b$ of the system. 


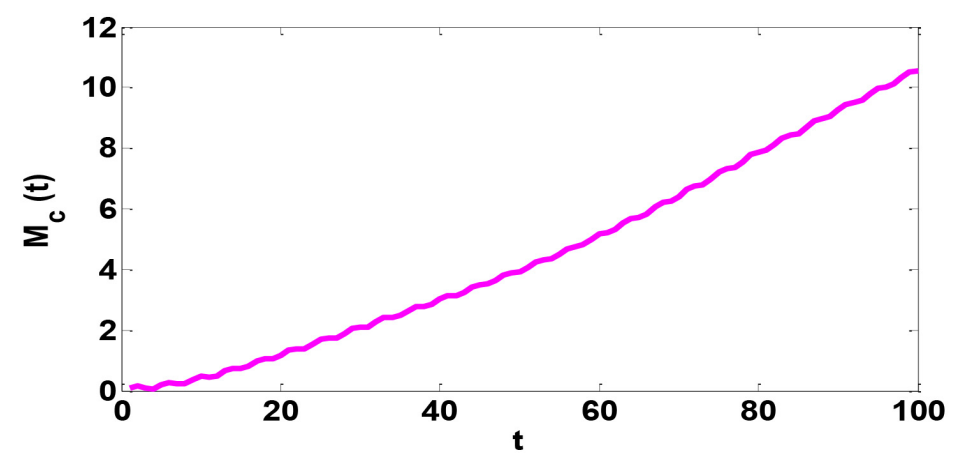

Figure 6. $M_{c}(t)$ versus time $t$ for system (1).

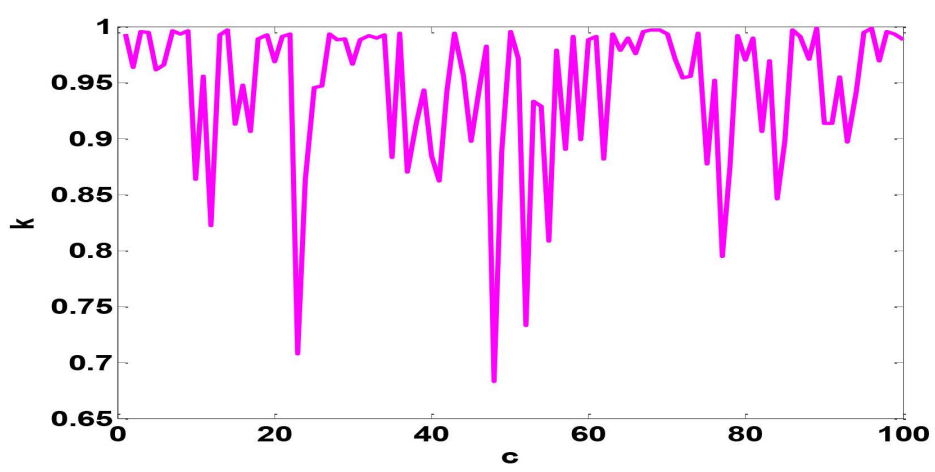

Figure 7. $k$ versus $c$ for system (1).

By adding the control units to the system, we get:-

$$
\left\{\begin{array}{l}
x_{t+1}=x_{t}+y_{t}+u_{1} \\
y_{t+1}=0.1 x_{t}^{2}+0.1+y_{t}\left(-x_{t}-b y_{t}\right)+y_{t}+u_{2}
\end{array}\right.
$$

where $u_{1}, u_{2}$ are the controllers for the adaptive feeding, defined as follows:-

$$
\left\{\begin{array}{l}
u_{1}=-x_{t}-y_{t}-M_{1} x_{t} \\
u_{2}=-0.1 x_{t}^{2}-0.1+x_{t} y_{t}+\beta y_{t}^{2}-y_{t}-M_{1} y_{t}
\end{array}\right.
$$

where $\beta$ is an approximate parameter of $b$ and $M_{1}, M_{2}$ are positive constants by substituting (9) in (8) we get:

$$
\left\{\begin{array}{l}
x_{t+1}=-M_{1} x_{t} \\
y_{t+1}=(-b+\beta) y_{t}^{2}-M_{2} y_{t}
\end{array}\right.
$$

Let the error for the discretionary parameter as follows:

$$
e_{b}=-b+\beta
$$

By substituting (11) into (10):

$$
\left\{\begin{array}{l}
x_{t+1}=-M_{1} x_{t} \\
y_{t+1}=e_{b} y_{t}^{2}-M_{2} y_{t}
\end{array}\right.
$$

\subsection{Numerical Results of System (10)}

In this section we will stability test of fixed points $p_{0}, p_{1}$ for system (1) in sys- 
tem (10), where $M_{1}=0.7, M_{2}=0.5$ and parameter $\beta$ is an estimated parameter of $b$ which is estimated at $=2.1$.

\subsubsection{Characteristic Equation Roots Test}

To study the stability of fixed points in System (1), using the characteristic equation root test, we will find the Jacobian Matrix of System (10) as follows:

$$
J_{\left(x_{t}, y_{t}\right)}=\left[\begin{array}{cc}
-M_{1} & 0 \\
0 & 2(-b+\beta) y_{t}-M_{2}
\end{array}\right]
$$

Substituting fixed point $p_{0}=(i, 0)$ and the values of $M_{1}, M_{2}$, into Equation (13) we get:-

$$
J_{(0,0)}=\left[\begin{array}{cc}
-0.7 & 0 \\
0 & -0.5
\end{array}\right]
$$

Finding the determinant ( $\operatorname{Det}(\lambda I-J)=0$ ) we obtain the following equation:-

$$
\lambda^{2}+1.2 \lambda+0.35=0
$$

By analyzing the quadratic Equation (14), and Proposition (1) we get:-

$$
\left|\lambda_{1}\right|=0.5,\left|\lambda_{2}\right|=0.7
$$

Therefore, the point $p_{0}$ is stable. Since point $p_{1}$ is symmetric to point $p_{0}$, it has the same results, that is, it is also a stable point, which leads to the system (9) being a stable system.

\subsubsection{Jury Test}

After the adaptive control technique of system was carried out and the characteristic equation roots were tested for the points in the controlled system (10), distinct equations were obtained with the values of their real coefficients, and thus it became possible for us to use a jury test to study the stability of fixed points in system (10). The coefficients of characteristic Equation (14) are:

$$
a_{0}=0.35, a_{1}=1.2, a_{2}=1
$$

Accordingly, we form the Jury test Table 3 for point $p_{0}$ as follows:

Since all of the conditions for Jury test for point $p_{0}$ are satisfies Proposition (2), then point $p_{0}$ is stable, and point $p_{1}$ is stable too, which leads to the system (10) is stable.

\subsection{Lyapunov Exponent of System (10)}

In this section we will use Lyapunov exponent test to study the chaotic behavior of

Table 3. Table of Jury test for point $p_{0}$ in the system (10).

\begin{tabular}{ccc}
\hline$\lambda^{0}$ & $\lambda^{1}$ & $\lambda^{2}$ \\
\hline 0.35 & 1.2 & 1 \\
1 & 1.2 & 0.35 \\
-0.8775 & -0.78 & \\
-0.78 & -0.8775 & \\
0.1616 & &
\end{tabular}




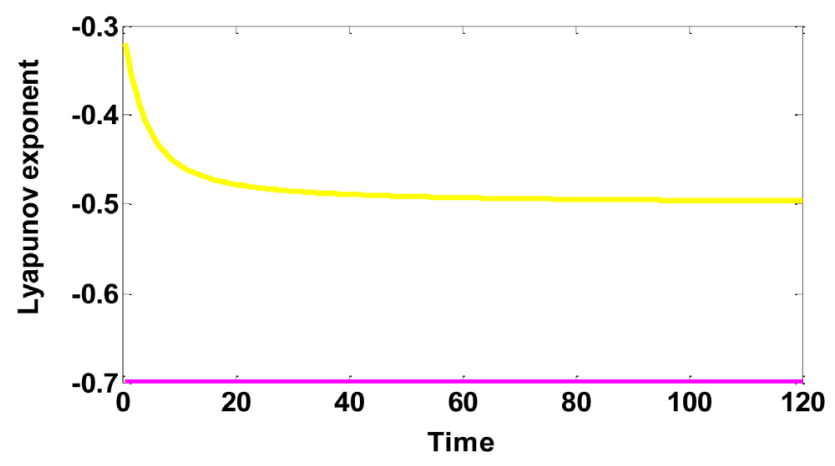

Figure 8. Lyapunov exponent of system (10).

system (10), and by using a written program in MATLAB, we obtained:-

$$
L_{1}=-0.700000, L_{2}=-0.418868
$$

And since all the obtained values are negative values, this means that system (10) is a regular system as shown in Figure 8.

\section{Conclusion}

In this paper, the stability and chaos of a two-dimensional discrete time dynamical system with hidden attractors was studied, and the fixed points were found: $p_{0}=(i, 0), p_{1}=(-i, 0)$ which shows us that the points are complex. The stability analysis of fixed points was analyzed by using the characteristic equation roots test, which shows us that the points are non-hyperbolic points, so we could not use jury test for the stability, the dynamic behavior of system was analyzed and a time series was generated for each state of system and phase space and bifurcation diagrams of the system were found. Chaos diagnosis in the system by using Lyapunov exponent test and it was obtained: $L_{1}=-0.250000, L_{2}=1.853981$. Lyapunov dimension: $D_{L}=0.865156$, and from the binary test $(0-1)$ we get the index value $k=0.9305$, which is close to one, all show that the system (1) is chaotic. The adaptive control technique of the chaotic system was carried out. Stability tests: roots of the characteristic equation and jury test for points in the controlling system (10), and Lyapunov exponent test obtaining the values:

$L_{1}=-0.700000, L_{2}=-0.41886$, which all show that the system after control is stable and regular.

\section{Acknowledgements}

The authors acknowledge the support university of Mosul and college of computer science and mathematics.

\section{Conflicts of Interest}

The authors declare no conflicts of interest regarding the publication of this paper.

\section{References}

[1] Lorenz, E.N. (1963) Deterministic Nonperiodic Flow. Journal of Atmospheric Sciences, 
20, 130-141.

https://doi.org/10.1175/1520-0469(1963)020\%3C0130:DNF\%3E2.0.CO;2

[2] Zhang, S. and Zeng, Y. (2019) A Simple Jerk-Like System without Equilibrium: Asymmetric Coexisting Hidden Attractors, Bursting Oscillation and Double Full Feigenbaum Remerging Trees. Chaos, Solitons \& Fractals, 120, 25-40. https://doi.org/10.1016/j.chaos.2018.12.036

[3] Jafari, S., Sprott, J.C. and Mohammad Reza Hashemi Golpayegani, S. (2013) Elementary Chaotic Flows with No Equilibria. Physics Letters A, 377, 699-702. https://doi.org/10.1016/j.physleta.2013.01.009

[4] Leonov, G.A. and Kuznetsov, N.V. (2015) On Differences and Similarities in the Analysis of Lorenz, Chen, and Lu Systems. Applied Mathematics and Computation, 256, 334-343. https://doi.org/10.1016/j.amc.2014.12.132

[5] Leonov, G.A. (2012) General Existence Conditions of Homoclinic Trajectories in Dissipative Systems. Lorenz, Shimizu-Morioka, Lu and Chen Systems. Physics Letters $A$, 376, 3045-3050. https://doi.org/10.1016/j.physleta.2012.07.003

[6] Jafari, S., Sprott, J.C. and Nazarimehr, F. (2015) Recent New Examples of Hidden Attractors. The European Physical Journal Special Topics, 224, 1469-1476. https://doi.org/10.1140/epjst/e2015-02472-1

[7] Jafari, S. and Sprott, J.C. (2013) Simple Chaotic Flows with a Line Equilibrium. Chaos, Solitons \& Fractals, 57, 79-84. https://doi.org/10.1016/j.chaos.2013.08.018

[8] Wei, Z., Wang, R. and Liu, A. (2014) A New Finding of the Existence of Hidden Hyperchaotic Attractors with No Equilibria. Mathematics and Computers in Simulation, 100, 13-23. https://doi.org/10.1016/j.matcom.2014.01.001

[9] Volos, C.K., Jafari, S., Kengne, J., Munoz-Pacheco, J.M. and Rajagopal, K. (2019) Nonlinear Dynamics and Entropy of Complex Systems with Hidden and Self-Excited Attractors. Entropy, 21, Article No. 370. https://doi.org/10.3390/e21040370

[10] Joseph, J. and Ivan, A. (1990) Feedback and Control Systems. McGraw-Hill, New York.

[11] Aziz, M.M. (2018) Stability Analysis of Mathematical Model. International Journal of Science and Research, 7, 147-148.

[12] Singh, H., Dhar, J. and Bhatti, H.S. (2015) Discrete-Time Bifurcation Behavior of a Prey-Predator System with Generalized Predator. Advances in Difference Equations, 2015, Article No. 206. https://doi.org/10.1186/s13662-015-0546-Z

[13] Xin, B. and Li, Y. (2013) Bifurcation and Chaos in a Price Game of Irrigation Water in a Coastal Irrigation District. Discrete Dynamics in Nature and Society, 2013, Article ID: 408904. https://doi.org/10.1155/2013/408904

[14] Vaidyanathan, S. (2016) A Novel 3-D Jerk Chaotic System with Three Quadratic Nonlinearities and Its Adaptive Control. Archives of Control Sciences, 26, 19-47. https://doi.org/10.1515/acsc-2016-0002

[15] Layek, G.C. (2015) An Introduction to Dynamical Systems and Chaos. Springer, New Delhi. https://doi.org/10.1007/978-81-322-2556-0

[16] Aziz, M.M. and Faraj, M.N. (2012) Numerical and Chaotic Analysis of CHUA'S CIRCUT. Journal of Emerging Trends in Computing and Information Sciences, 3, 783-791.

[17] Gottwald, G.A. and Melbourne, I. (2009) On the Implementation of the 0-1 Test for Chaos. Xulvi-Brunet. SIAM Journal on Applied Dynamical Systems, 8, 129-145. https://doi.org/10.1137/080718851

[18] Aziz, M.M. and Jihad, O.M. (2021) Stability, Chaos Diagnose and Adaptive Control 
of Two Dimensional Discrete-Time Dynamical System. Open Access Library Journal, 8, Article No. e7270. https://doi.org/10.4236/oalib.1107270

[19] Aziz, M.M. and Merie, D.M. (2020) Stability and Adaptive Control with Synchronization of 3-D Dynamical System. Open Access Library Journal, 7, Article No. e6075. https://doi.org/10.4236/oalib.1106075

[20] Panahi, S., Sprott, J.C. and Jafari, S. (2018) Two Simplest Quadratic Chaotic Maps without Equilibrium. International Journal of Bifurcation and Chaos, 28, Article ID: 1850144. https://doi.org/10.1142/S0218127418501444

[21] Ouannas, A., Khennaoui, A.A., Momani, S., Grassi, G., Pham, V.T., ElKhazali, R. and Vo Hoang, D. (2020) A Quadratic Fractional Map without Equilibria: Bifurcation, 0-1 Test, Complexity, Entropy, and Control. Electronics, 9, Article No. 748. https://doi.org/10.3390/electronics 9050748 